Unter der Ocrelizumab-Therapie kommt es zu einer signifikanten Abnahme der jährlichen Schubrate (unter Ocrelizumab 600 mg: $80 \%, p=0,0005$; unter Ocrelizumab $2.000 \mathrm{mg}: 73 \%, \mathrm{p}=0,0014)$ und der gadoliniumanreichernden Läsionen (nach 24 Wochen: unter $600 \mathrm{mg}$ Ocrelizumab um $89 \%, \mathrm{p}<0,0001$, unter $2.000 \mathrm{mg}$ um $96 \%$ geringer als unter Placebo, $p<0,0001$ ), so die Ergebnisse einer Phase-II-Studie [Kappos L et al. Lancet 2011; 378: 1779-87]. Tackenberg betonte, er sei überrascht, dass die MRT-Ergebnisse so eindeutig seien.

Dr. Friederike Holthausen

Pressekonferenz „B-focused - Die B-Zelle als Target bei der Multiplen Sklerose", Düsseldorf; Veranstalter: Roche Pharma

\title{
Adulte ADHS: Psychopathologische Symptomatik effizient eindämmen
}

\begin{abstract}
— Die Diagnose der ADHS im Erwachsenenalter wird anhand der diagnostischen Kriterien des DSM-5 gestellt. Erschwert wird sie mitunter durch die begrenzte Introspektionsfähigkeit der Betroffenen, die retrospektive Erfassung von ADHS-Symptomen in der Kindheit sowie die oftmals schwierige Abgrenzung zu einer Persönlichkeitsstörung mit Beeinträchtigung der Impulskontrolle oder zur Borderline-Persönlichkeitsstörung. Laut Professor Michael Rösler vom Neurozentrum des Universitätsklinikums des Saarlandes werden vier Subtypen unterschieden:

_ ADHS mit überwiegender Aufmerksamkeitsstörung

_ ADHS mit überwiegender Hyperaktivität und Impulsivität

_ Kombinierte ADHS

_ ADHS in partieller Remission
\end{abstract}

Für die Diagnose nach DSM 5 müssen neben mindestens fünf der neun Kriterien für Aufmerksamkeitsstörungen auch fünf von neun Kriterien aus dem Bereich Hyperaktivität und Impulsivität nachgewiesen werden. Damit liegt die diagnostische Schwelle etwas niedriger als beim DSM-IV. Erleichtert wird die Diagnostik durch die Veränderung des Alterskriteriums für den Nachweis der Psychopathologie: Die Symptomatik muss spätestens im zwölften Lebensjahr nachweisbar sein. Mit dem Diagnosetool IDA-R steht ein Instrument mit Selbstbeurteilungsund Fremdbeurteilungsskalen zur Verfügung, das den diagnostischen Prozess systematisch abarbeitet.

In der medikamentösen Behandlung der adulten ADHS ist gemäß den evidenzbasierten Leitlinien Methylphenidat (MPH) das Mittel der ersten Wahl. Als Mittel der zweiten Wahl kommt Atomoxetin in Be- tracht. Rösler stellte die naturalistische Beobachtungsstudie IDEA vor [Fischer $\mathrm{R}$ et al. Poster, $5^{\text {th }}$ World Congress on ADHD 2015, Glasgow/Scotland], die nach seinen Worten ähnliche Behandlungsergebnisse erbrachte wie die früheren verblindeten und kontrollierten Studien [Rösler M et al. Eur Arch Psychiatry Clin Neurosci 2009; 259 (2): 120 - 9; Retz W et al. World J Biol Psychiatry 2010; 11 (5): 709-18]. Danach reduziert MPH in retardierter Form (Medikinet ${ }^{\circledR}$ adult) die klassische psychopathologische Symptomatik der ADHS und weitere assoziierte Phänomene wie emotionale Dysregulation.

In IDEA wurden 468 Erwachsene mit ADHS (58\% Männer) auf retardiertes MPH erstoder neu eingestellt und über 3,3 $\pm 1,6$ Monate beobachtet. Bei einem Viertel der Patienten war die ADHS bereits in der Kindheit diagnostiziert worden. Fast drei Viertel der Patienten ging es gemäß Arzturteil bei der zweiten Visite "viel besser" oder "sehr viel besser". Rösler betonte, dass wegen möglicher Nebenwirkungen regelmäßige Kontrolluntersuchungen vor und während der Behandlung unverzichtbar seien. Typische Nebenwirkungen unter $\mathrm{MPH}-$ Therapie sind Appetitminderung, Übelkeit, Erbrechen, Gewichtsverlust, Blutdruckerhöhung, Pulsbeschleunigung, Herzklopfen und Kopfschmerzen. Diese Nebenwirkungen treten in der Regel in der Aufdosierungsphase auf, langfristige schädliche Wirkungen seien nicht bekannt, so Rösler.

\section{Dr. Gunter Freese}

Pressekonferenz "Meet the experts - MPH, effektive Symptomkontrolle und Titration", im Rahmen des DGPPN-Kongresses, Berlin, 26.11.2015; Veranstalter: MEDICE Arzneimittel

\section{G-BA-Beschluss zur Nutzenbewertung}

Der Gemeinsame Bundesausschuss (GBA) hat dem Faktor-Xa-Inhibitor Edoxaban (Lixiana ${ }^{\circledR}$ ) am 21. Januar 2016 einen Hinweis auf einen geringen Zusatznutzen in der Prophylaxe von Schlaganfällen und systemischen embolischen Ereignissen (SEE) bei Patienten mit nicht valvulärem Vorhofflimmern (nvVHF) attestiert. Ausschlaggebend für diese Bewertung waren vor allem die signifikante Reduktion hämorrhagischer Schlaganfälle sowie die signifikante Reduktion schwerer und klinisch relevanter nicht schwerer Blutungen unter Edoxaban im Vergleich zur zweckmäßigen Vergleichstherapie mit dem Vitamin-K-Antagonisten Warfarin. Edoxaban ist damit das einzige einmal tägliche orale nicht VKA-Antikoagulanz (NOAK) mit bescheinigtem Zusatznutzen. Grundlage für die Entscheidung des G-BA waren die Daten der Zulassungsstudie ENGAGE AF-TIMI 48.

Nach Information von Daiichi Sankyo

\section{Zulassungserweiterung}

IncobotulinumtoxinA (Xeomin ${ }^{\oplus}$ ) ist von der US-amerikanischen Zulassungsbehörde FDA zur Behandlung der Spastik der oberen Extremitäten bei Erwachsenen ohne Einschränkung der Ätiologie zugelassen worden.

Nach Informationen von Merz

\section{Neuer Name - neuer Vertrieb}

Der Arzneimittelhersteller Servier und die Gaia AG haben sich auf eine Vertriebskooperation für das Onlinetherapieprogramm "deprexis" geeinigt. Das Produkt wird seit November 2015 unter dem neuen Namen deprexis ${ }^{\circledR} 24$ von Servier Deutschland vertrieben.

Nach Informationen von Servier

\section{Spezifisches Antidot für den Notfall}

Nach der Zulassung durch die EMA steht Praxbind ${ }^{\circledR}$, das Pradaxa ${ }^{\circledR}$-spezifische Antidot, jetzt deutschlandweit bereits in vielen Notfallambulanzen zur Verfügung.

Nach Informationen von Boehringer Ingelheim 